\title{
Editorial
}

\section{Themes to be better?}

Dear readers, in response to requests from those that contribute regularly to the Journal we will endeavour to include a number of thematic issues each year. If these are well received then proposed themes will be advertised and suggestions for themes will be canvassed. Presently two themes have been proposed, neuroimaging and bipolar disorders. The latter is the focus of this issue.

Over the past decade bipolar disorders have attracted widespread attention with investment from research funding agencies and government bodies. Societies such as the International Society for Bipolar Disorders (ISBD), organisations and consumer groups have formed around the disorder. These have spawned conferences such as the International Conference on Bipolar Disorder (ICBD) that is held every two years in Pittsburgh, and many other meetings that have a particular focus on bipolar disorder. Suffice to say there has been a wealth of activity. This is reflected in the research that has been conducted both with respect to the depth and breadth of studies as demonstrated by the submissions published in this issue of Acta Neuropsychiatrica.

For example, Colom's group (1) report on valuable 5 year follow-up data concerning the response to psychoeducation in bipolar patients. Scheuch et al. (2) investigate emotional vulnerability and cognitive regulation both in patients with the illness and their healthy siblings. Gomes and colleagues (3) address the important issue of suicide and how this may be related to physical health. Chengappa and colleagues (4) report on the effects of risperidone administered as a long-acting injection as compared to oral atypical antipsychotic medication in the treatment of bipolar disorder. The sub-theme of antipsychotics continues in the Comment and Critique section with observations from Byrne and colleagues (5) involving families of young people taking antipsychotic medication. The bipolar theme is further addressed in this section with a validity report concerning the Portuguese version of the Bipolar Depression Rating Scale from Magalhaes et al. (6). Finally, even one of our regular sections namely, Intervention Insights, addresses the growing problem of alcohol and drug abuse in patients with bipolar disorder and those with schizophrenia (7).

These interesting papers and commentaries provide a rich and diverse overview of various aspects of bipolar disorder however, despite many research efforts the definition of the illness continues to be a major stumbling block. This is critical and is no doubt a focus of DSM V discussions.

From a neuropsychiatric perspective bipolar disorder provides an insight into emotional regulation (dysregulation). If pursued longitudinally it allows potential for within group comparison and the distinction of trait and state markers. With the potential emergence of biomarkers those investigating bipolar disorder, in addition to better identifying the illness, may also be able to provide interesting insights into emotion generation and modulation in health.

\section{Professor Gin S. Malhi \\ Sydney Medical School, University of Sydney, Sydney, Australia}

\section{References}

1. Colom $\mathrm{F}$ et al. Has number of previous episodes any effect on response to group psychoeducation in bipolar patients? A 5-year follow-up post-hoc analysis. Acta Neuropsychiatr 2010;22:50-53.

2. SCHEUCH K et al. Emotional vulnerability and cognitive control in patients with bipolar disorder and their healthy siblings: a pilot study. Acta Neuropsychiatr 2010;22:54-62.

3. Gomes F et al. Obesity is associated with previous suicide attempts in bipolar disorder. Acta Neuropsychiatr 2010;22:63-67.

4. Chengappa KnR et al. A Pilot, 15-month, randomized effectiveness trial of Risperidone long-acting injection (RLAI) versus oral atypical antipsychotic agents (AAP) in persons with bipolar disorder. Acta Neuropsychiatr 2010;22:68-80.

5. BYRne $\mathrm{S}$ et al. Observations from postal research involving families of young people taking antipsychotic medication. Acta Neuropsychiatr 2010;22:102.

6. Magalhaes P. Validity of the Portuguese version of the Bipolar Depression Rating Scale. Acta Neuropsychiatr 2010;22:100-101.

7. Lubman D, Berk M. Pharmacotherapy for co-occurring alcohol and drug disorders in schizophrenia and bipolar disorder: where is the evidence? Acta Neuropsychiatr 2010;22:95-97. 